\title{
A132 consecuencias personales en LA www.aibr.org RUPTURA DE LA VIDA LABORAL. EL CASO DE TELEFÓNICA ${ }^{1}$
}

\section{Ma Victoria Inmaculada Velasco Juez}

\author{
Licenciada en Psicología (UCM). DEA en Antropología Social (UCM). E-mail:
} inma velasco@telefonica.net .

\section{Resumen}

Desde mediados de los años setenta, el mundo del trabajo de los países occidentales comienza a cambiar progresivamente en su estructura y fisonomía. En los últimos años, como consecuencia de la búsqueda de competitividad y la política de fusiones y adquisiciones llevada a cabo en España por algunas grandes empresas, han aumentado de forma significativa las reducciones de plantilla. Considerando que estamos ante un proceso cultural de las sociedades contemporáneas y siendo parte integrante de ese colectivo, cada día más numeroso, de personas desvinculadas del trabajo remunerado a una edad temprana, estudié qué supone este hecho para un grupo de trabajadores de Telefónica. Esta empresa está llevando a cabo la mayor regulación de empleo de nuestro país; terminará 2007, por un plan iniciado en 1993, con una plantilla reducida en un $66 \%$. Para analizar cómo afecta este hecho a los trabajadores y en qué ámbitos, aspectos y dimensiones de su vida, tuve en cuenta las diferencias de interpretación de sus experiencias en cuanto al tiempo, al espacio y a la sociabilidad. También consideré las diferencias individuales: género, situación familiar, formación, trayectoria laboral, puesto que se ocupaba en la empresa, estrategias de ocupación del tiempo de ocio, etc. A partir de la etnografía se concluye que, siendo la actividad laboral remunerada un factor de identidad personal y social, un factor de integración y estructuración del ser humano, desde una perspectiva antropológico-social, la ruptura de dicha trayectoria, a una edad temprana y de forma brusca y no deseada, conlleva la necesidad de ajuste en todas las actividades del ser humano.

\section{Palabras clave}

Desvinculación temprana, trayectoria laboral, ocupación del tiempo, entorno relacional, salud emocional.

\begin{abstract}
Since the mid seventies, labour in Western Countries has progressively changed its structure and appearance. In the last years, as a result of competitiveness and the policy of fusions and acquisitions carried out in Spain by some great firms, layoffs have become significantly frequent. Considering that there exists a growing up cultural process in contemporary societies, related to the people who has to leave the paid work in an earlier age, I studied the implications of this circumstance in a group of employees of the company Telefónica. This firm
\end{abstract}

\footnotetext{
${ }^{1}$ Este artículo procede de la investigación "Análisis antropológico-social de la desvinculación temprana del trabajo remunerado en empleados de Telefónica. Efectos en la salud y en las relaciones familiares y sociales", que llevé a cabo para la obtención del DEA, Departamento de Antropología Social, Facultad de Ciencias Políticas y Sociología, Universidad Complutense de
} Madrid. Curso 2004-2005. 
has carried out the biggest employment adjustment in our country, that will finished in 2007, after a process that started on 1993 . The total reduced staff will be $66 \%$. In order to analyse the impact on employees and its different aspects and life dimensions, I took into account their different interpretation of experiences related to time, space and sociability. I also considered individual differences: gender, family situation, education, job experience, position in the company, strategies of time occupation, leisure, etc. From the ethnography I may conclude that, being paid job activity a personal identity and social factor, an integration and human structural factor, from an anthropological-social perspective; the break down of such trajectory in an early age and in a non-wished manner, involves the need of the adjustment on every activity of the human being.

\section{Keywords}

Early retirement, laboral career, lifetime schedule, social environment, emotional health.

\section{Introducción}

$\mathrm{E}_{\mathrm{s}}$ propósito al iniciar este trabajo era estudiar, desde una perspectiva antropológico-social, la situación de las personas que ven interrumpida, bruscamente y a edad temprana su actividad laboral remunerada, por motivos totalmente ajenos a su deseo personal. Este hecho, conocido como prejubilación o desvinculación temprana del trabajo remunerado, ha sido el cauce que han utilizado gran número de empresas para reducir sus plantillas.

En el ordenamiento jurídico español no existe el concepto de prejubilación, si bien se contemplan situaciones que pueden asimilarse a él. Expondremos, sin embargo, la definición que propone el Consejo Económico y Social (CES):

[...] cabría considerar prejubilación la situación en que se encuentra un trabajador de edad avanzada y en razón de tal circunstancia, cuando se extingue su relación laboral sin expectativas de reingreso a la vida laboral activa y hasta el momento en que pase a percibir una pensión de jubilación, ya sea anticipada u ordinaria. (CES, 2000: 40-41) ${ }^{2}$

En cuanto a la desvinculación, tampoco me consta una definición oficial del término. Es utilizado, en sustitución de 'prejubilación', en el Plan Social ${ }^{3}$ 2003-2007 del Convenio Colectivo de Telefónica de España, S.A.U. (a partir de ahora Telefónica) firmado el día 29 de Julio de 2003. Según explicación dada por un sindicalista de la Unión General de Trabajadores (UGT) de Telefónica, este cambio se produjo, a instancias del Gobierno de España en vigor en ese momento, con el fin de que el Expediente de Regulación de Empleo (ERE) que Telefónica pretendía presentar no fuera rechazado. La connotación principal de este término es que el aporte a la Seguridad Social, cuando el trabajador cesa en su actividad laboral a través de un ERE, corre a cargo de la empresa y no del Estado, como ocurría en el caso de las, hasta entonces, 'prejubilaciones'.

\footnotetext{
${ }^{2}$ CES (2000). Informe sobre vida laboral y prejubilaciones. Madrid.

${ }^{3}$ El Plan Social es el documento que recoge las condiciones de aplicación del ERE (2003-2007) y es un anexo al Convenio
} Colectivo 2003-2005 de Telefónica. 
Otro concepto que conviene tener claro es el de jubilación anticipada. Es la situación en la que un trabajador es pensionista de la Seguridad Social con una edad inferior ${ }^{4}$ a los 65 años, que es la establecida en España para la finalización de la vida laboral. Los trabajadores que se jubilan anticipadamente sufren una penalización en su pensión, para el resto de su vida, de entre un 24 y un $40 \%{ }^{5}$, siempre que hayan cotizado un mínimo de 40 años, y de mayor cuantía si han cotizado menos.

Pero, ¿por qué nos ocupamos de este tema?, ¿qué pasa en el mundo laboral? Desde mediados de los años setenta el mundo del trabajo de los países occidentales comienza a cambiar progresivamente su propia estructura y fisonomía. El modo de producción taylorista-fordista, con la estandarización de los productos, las nuevas técnicas de fabricación, las necesidades y demandas de los consumidores, la especialización de las tareas laborales, la sincronización de las fases y los tiempos de producción y la maximización de las dimensiones de la dirección organizativa de las empresas, comienza a encontrar serias dificultades, tanto en el marco nacional como en el internacional. Tiene lugar así el tránsito hacia la sociedad postindustrial o postfordista. En este escenario, las empresas de los mayores países occidentales registran en el plano productivo, a finales de los 70 y durante toda la década de los 80 , profundas crisis y procesos de reestructuración, que lenta pero inexorablemente modifican su naturaleza y características. Se van instaurando nuevos modos productivos basados en la flexibilidad, tanto numérica, como salarial y funcional, y van acompañados con frecuencia de una creciente desregulación del mercado de trabajo.

En España, ya en la Constitución ${ }^{6}$ de 1978, la productividad queda erigida en principio incuestionable sobre el que deben descansar las demás consideraciones. Previamente, en los Pactos de la Moncloa de 1977, se acordó la posibilidad de hacer contratos temporales ${ }^{7}$ y el derecho de los empresarios al despido de hasta un $5 \%$ de la plantilla. En 1984 la reforma del Estatuto de los Trabajadores, que declaraba conseguir "la eliminación de las restricciones de la contratación temporal", constituye el mejor ejemplo de cómo la legislación laboral se está adecuando a la práctica empresarial de gestión del trabajo. Con esta reforma se amplían las causas de despido y se simplifican los mecanismos de Expedientes de Regulación de Empleo, en cuya tramitación los sindicatos pierden peso.

Los flujos de salida del mercado de trabajo pueden ser de dos tipos: individuales y colectivos. Hago hincapié en este segundo por ser el que afecta al grupo de trabajadores de Telefónica que he estudiado. La Ley de Estatuto de los Trabajadores ${ }^{8}$ dice que

\footnotetext{
${ }^{4}$ Con 61 años como norma general o con 60 si el trabajador había cotizado antes del 1 de enero de 1967, según el actual Régimen General de la Seguridad Social. Ley 47/1998, de 23 de diciembre.

${ }^{5}$ Entre un 6 y un $8 \%$ por año que adelantan la jubilación, según que el paso a esa situación haya sido impuesto o voluntario.

6 "Se reconoce la libertad de empresa en el marco de la economía de mercado. Los poderes públicos garantizan y protegen su ejercicio y de la defensa de la productividad, de acuerdo con las exigencias de la economía general y, en su caso, de la planificación". Constitución Española (1978). Artículo 38

7 "Al objeto de fomentar el empleo juvenil se autoriza, dentro de un programa experimental que se concretará, la contratación temporal por un plazo máximo de dos años para los empleos derivados de los nuevos puestos de trabajo que se creen a partir del 1 de noviembre de 1977, siempre que los mismos se cubran con personas que desempeñen por vez primera un trabajo". Pactos de la Moncloa (Apartado I, E, 2). Octubre de 1977.

${ }^{8}$ Ley 8/1980, de 10 de marzo, del Estatuto de los Trabajadores. Artículo 51.
} 
Se entenderá por despido colectivo la extinción de contratos de trabajo fundada en causas económicas, técnicas, organizativas o de producción, cuando, en un período de noventa días, la extinción afecte al menos a: a) Diez trabajadores, en las empresas que ocupen menos de cien trabajadores. b) El 10 por 100 del número de trabajadores de la empresa en aquellas que ocupen entre cien y trescientos trabajadores. c) Treinta trabajadores en las empresas que ocupen trescientos o más trabajadores. (Estatuto de los Trabajadores, 1980, Artículo 51).

Según esta misma Ley, "el empresario que tenga intención de efectuar un despido colectivo deberá solicitar autorización para la extinción de los contratos de trabajo conforme al procedimiento de regulación de empleo previsto en esta Ley y en sus normas de desarrollo reglamentario" (Estatuto de Ios Trabajadores, 1980: 51). El procedimiento, el ERE, se inicia mediante la solicitud a la autoridad laboral competente y la apertura simultánea de un periodo de consultas con los representantes legales de los trabajadores. Puede iniciarlo el empresario interesado o los trabajadores a través de sus representantes legales, si se presumiera que la no incoación del mismo por el empresario pudiera ocasionarles perjuicio de imposible o difícil reparación.

En los últimos años, como consecuencia de la búsqueda de competitividad y la política de fusiones y adquisiciones llevada a cabo en España por algunas grandes empresas, como Telefónica, RENFE y algunos Bancos, han aumentado de forma significativa las reducciones de plantillas de forma pactada, como las desvinculaciones y las jubilaciones anticipadas, que han llegado a afectar incluso a personas con menos de 50 años. Se empezó por ofrecer jubilaciones anticipadas a los 60 años, se siguió con desvinculaciones desde los 55 y en la actualidad es frecuente que con 50 se interrumpa la vida laboral, y hay casos, aunque excepcionales, en los que si no es por un ERE, es, como nos dice Ana Ma Rivas (2005) en una reciente investigación, por un Expediente de Movilidad Geográfica (EMG), por el que los trabajadores y trabajadoras ven finalizada su vida laboral.

\begin{tabular}{|c|c|c|c|c|c|}
\hline AÑO & $\mathbf{2 0 0 1}$ & $\mathbf{2 0 0 2}$ & $\mathbf{2 0 0 3}$ & $\mathbf{2 0 0 4}$ & $\mathbf{2 0 0 5}$ \\
\hline TOTAL & 132,313 & 86,510 & 97,885 & 76,349 & 85,468 \\
\hline
\end{tabular}

TABLA 1. Expedientes de Regulación de Empleo. Trabajadores afectados. (Fuente: Ministerio de Trabajo y Asuntos Sociales; Fecha: Abril de 2006)

Este hecho, que afecta a los trabajadores de más edad, es relativamente nuevo, lo que justifica que no haya muchas investigaciones al respecto. Es pionero en España el estudio sobre prejubilaciones, jubilaciones anticipadas y otras formas de expulsión de los trabajadores mayores del mercado de trabajo, llevado a cabo por J. M. Riera (1999). Este autor pone de manifiesto que la posibilidad de cesar en la actividad laboral a los 50 años no es un fenómeno coyuntural, asociado a situaciones de crisis o recesión, sino un fenómeno estructural. Esta es también la opinión del CES:

La salida del empleo de colectivos de trabajadores con una característica común, superar una determinada edad, ha respondido en el tiempo a causas o circunstancias de diferente tenor. En las décadas de 1970 y 1980 este fenómeno tenía su causa en situaciones concretas de reconversión o crisis empresariales. En la actualidad, el riesgo de expulsión del mercado laboral de trabajadores de edad avanzada adquiere un perfil más estructural, independizándose, en parte, de los avatares negativos que puedan afectar a las empresas o a los sectores. (CES, 2000: 40). 
La presente investigación se llevó a cabo en Telefónica, empresa pionera en el sector de las Telecomunicaciones y emblemática en la llamada 'sociedad de la información'. Es una empresa muy representativa en el ámbito que pretendía estudiar. El acelerado proceso de innovación tecnológica era la razón irrevocable, según la empresa, para el importante ajuste de plantilla. Contaba con 74.340 empleados en 1993 y pretende cerrar 2007 con menos de 25.650 trabajadores. Son casi 50.000 empleos menos en 14 años, equivale a una reducción de más del $66 \%$ de su plantilla. Se trata de la mayor regulación de empleo llevada a cabo en España.

Este estudio pretende hacer una aportación, aún no existente en este tema, y es analizar, desde una perspectiva antropológica y social, qué supone la desvinculación temprana del trabajo remunerado para un grupo de trabajadores; en qué ámbitos, aspectos y dimensiones de su vida les afecta y cómo; y las diferencias de interpretación de sus experiencias y vivencias, en cuanto al tiempo, al espacio, a la sociabilidad... Todo ello teniendo en cuenta las diferencias individuales: género, situación familiar, formación, trayectoria laboral, puesto que se ocupaba en la empresa, estrategias de ocupación del tiempo de ocio, etc.

En los inicios de la década de los setenta tuvo lugar un proceso de innovación tecnológica sin precedentes, comenzó una etapa de grandes cambios en el sector de las telecomunicaciones que potenciaría su importancia estratégica para las economías contemporáneas y conllevaría una ruptura total con el pasado. Las innovaciones tecnológicas que han influido más en las telecomunicaciones han sido la fibra óptica y la digitalización de la información. Las tecnologías vía satélite y las comunicaciones móviles, también tuvieron gran peso en este proceso. Basta una mirada rápida a nuestro entorno para constatar la gran cantidad de actividades de nuestra vida cotidiana en las que hacemos uso de esas nuevas tecnologías de la comunicación: telebanco, telecompra a través de internet, teletrabajo, nuevos usos de los teléfonos móviles (acceso a internet, correo electrónico, datos, imágenes, etc.), TV a través de la línea telefónica, transmisión por módem de cable y satélite digital, difusión a través de la web de todo tipo de información, etc.

La incorporación de las nuevas tecnologías a los procesos productivos afecta a un número cada vez mayor de personas e influye en distintos aspectos de su vida laboral: en las condiciones de trabajo, el contenido de las tareas, el salario, la formación, la salud, los ritmos de trabajo, los tiempos de descanso, etc. Las nuevas formas particulares de empleo: contratos de trabajo por tiempo limitado, trabajo provisional, trabajo de jornada reducida, contratos por obra o servicio, contratos en prácticas, etc., se asemejan más a las antiguas formas de contratación, previas a la generalización del salariado, de modo que el estatuto del trabajador pierde fuerza ante las imposiciones del trabajo. "Flexibilidad" fue una manera de denominar a esta necesidad de ajuste del trabajador moderno a su tarea (Castel, 1995: 404-407). Pero la flexibilidad no se reduce a la necesidad de ajustarse mecánicamente a una tarea puntual, sino que exige que el trabajador esté de inmediato disponible para responder a las fluctuaciones de la demanda. Las empresas responden a la gestión de flujo continuo, a la producción sobre pedido. Para cumplir estos objetivos adoptan la llamada 
flexibilización, y lo hacen en dos sentidos: por un lado se recurre a la subcontratación o externalización de servicios (flexibilidad externa) y por otro recurren a la formación de sus empleados con flexibilidad y polivalencia (flexibilidad interna).

En el primer caso, flexibilidad externa, las políticas de externalización de actividades, llevadas a cabo por las grandes empresas, les permiten dotar de mayor flexibilidad y capacidad de respuesta a las unidades de producción. Estas empresas se transforman en 'grupos', dando lugar a la empresa principal y a las secundarias. La empresa principal subcontrata servicios a las empresas de su grupo, de manera que son éstas, las empresas secundarias, las que contratan nuevos trabajadores, pero lo hacen en condiciones más precarias y sin derechos adquiridos, aplicando las nuevas formas particulares de empleo. Con esta forma de proceder de las empresas grupo, las plantillas de la principal, cuyos trabajadores disfrutan de mejor salario, contrato indefinido, beneficios sociales, etc., se verán reducidas en gran número. Como no les resulta difícil demostrar que no necesitan tanto personal para la actividad que desarrollan, puesto que la han derivado a las empresas secundarias, si presentan un Expediente de Regulación de Empleo (hago referencia a esta situación porque se repite con frecuencia en nuestro país y porque ha dado origen a este estudio), consiguen deshacerse de gran número de trabajadores de forma legal. En general, los afectados son los trabajadores de más edad, que son los que tienen más derechos adquiridos y les resultan más caros a las empresas.

En el segundo caso, en cuanto a la flexibilidad interna, las empresas recurren a la capacitación de sus empleados con el objetivo de que sean polivalentes, es decir estén preparados para desempeñar cualquier tipo de actividad y en cualquier momento que se les demande. Se pretende que se adapten, en definitiva, a los nuevos cambios tecnológicos. Tampoco es difícil ver, en esta forma de gestión de los recursos humanos, que solo los trabajadores y las trabajadoras mejor preparados podrán adaptarse a la nueva situación; el resto, los menos 'aptos', serán objeto de 'despido'. Como en el caso anterior, los trabajadores más afectados por estos ajustes de plantillas son los de más edad, bien porque no les consideran capaces de adaptarse a las nuevas tecnologías, bien porque a las empresas les resulta más barato contratar trabajadores más jóvenes, con más formación, en general, más adaptables a las nuevas necesidades de la empresa y con menos derechos adquiridos $\mathrm{El}$ resultado es la invalidación de los 'trabajadores que envejecen' (pero a menudo tienen cincuenta años o menos), con demasiados años y no lo suficientemente formados para el reciclaje en nuevas tecnologías, pero demasiado jóvenes para jubilarse. Son trabajadores que ya no encuentran sitio en el proceso productivo, pero tampoco en otra parte (Castel, 1995).

\section{Aspectos metodológicos}

La investigación se desarrolló a partir de la perspectiva metodológica cualitativa. Fue una investigación etnográfica y se llevó a cabo mediante observación participante, entrevistas semiestructuradas y grupos de discusión. Se optó por entrevistas semiestructuradas, con el fin de poder abordar todos los ámbitos que consideraba imprescindibles para la investigación, y abiertas, de 
manera que cada sujeto entrevistado pudo aportar cualquier tipo de información que considerara importante en su vivencia personal. Con la entrevista no se buscaba información sobre hechos sino que se pretendía un acercamiento a los actores, de manera que, libremente, expresaran su sentimiento hacia la nueva situación que estaban viviendo, desde que Telefónica les había planteado, de forma personal o a través del Convenio Colectivo correspondiente, su salida de la empresa. Quería que el informante comentara este sentimiento, lo valorara, lo contrastara y lo relacionara; así pude saber cómo cada actor categorizaba su experiencia, sus creencias y sus valores en torno a la situación que estaba estudiando. Las entrevistas fueron precedidas de la exposición del objetivo general de la investigación y del objeto de las mismas. Los aspectos que consideré importantes fueron: la decisión, la trayectoria laboral, la salud, las actividades y las relaciones. Les planteé las preguntas de por qué, cómo y cuál era su sentimiento y valoración, tanto en el momento de la entrevista como en todas las etapas previas: propuesta de la situación por parte de la empresa, la toma de decisión, su vivencia posterior, etc. Además de su respuesta verbal, que fue grabada y posteriormente transcrita, tuve en cuenta la comunicación no verbal. Los silencios también eran importantes. En ocasiones, los informantes expresaban sentimientos, como si fueran de otras personas, erigiéndose en portavoces. Se puede justificar este hecho desde la Teoría de la Disonancia de Festinger (1957) ${ }^{9}$.

Los grupos de discusión surgieron a partir de los propios informantes. Desde el primer momento se detectó el interés, por parte de algunos de ellos, por hablar en grupo del tema que les planteaba, lo veían como una excusa para el reencuentro y pensaban en la oportunidad que tendrían para hablar de su nueva situación y de cómo la estaban abordando. En todos los casos fue uno de los informantes quien se encargó de reunir a un grupo, de entre cuatro y ocho afectados. Estos grupos me permitieron la observación del intercambio de experiencias y vivencias entre los actores; comprobé que facilitaban la consideración, en grupo, de algunos aspectos no relevantes para algunos de los sujetos, aunque sí para otros.

Realicé 23 entrevistas y se llevaron a cabo 6 grupos de discusión, tantos como consideré necesarios hasta la saturación de la información. Toda la información fue recogida entre los meses de enero y abril de 2005.

Los informantes fueron prejubilados y desvinculados, además de un caso de despido improcedente pactado; todos ellos residentes en Madrid y hacía entre uno y siete años que había finalizado su actividad laboral en la empresa. El límite mínimo de tiempo que debían llevar desvinculados para poder participar en el estudio era un año, se decidió al inicio de la investigación por considerar que si el tiempo transcurrido en la nueva situación era inferior, cabía la posibilidad de que los afectados la vivieran como una etapa de provisionalidad: vacaciones, días libres, baja de enfermedad, etc. Sin embargo, un año era tiempo suficiente para que el nuevo estatus fuera valorado como definitivo. Los

\footnotetext{
${ }^{9}$ Nos dice que una vez que un sujeto ha tomado una decisión, que ha sido objeto de una reflexión importante y que es crucial para su vida, éste se esfuerza en convencerse de que era lo mejor que podía hacer, de que la decisión tomada ha sido la correcta.

(c) Ma Victoria Inmaculada Velasco Juez. Publicado en AIBR. Revista de Antropología Iberoamericana, Ed. Electrónica

Vol 1. Num. 3. Agosto-Diciembre 2006. Pp. 465-486 Madrid: Antropólogos Iberoamericanos en Red. ISSN: 1578-9705
} 
informantes que llevaban más tiempo fuera de la empresa, se habían prejubilado hacía siete años, coincidiendo con el primer ajuste masivo de plantilla propuesto en el Convenio Colectivo de 1997 y que afectaba, por primera vez, a trabajadores desde los 55 años.

Las fuentes secundarias fueron las entidades que consideré disponían de la información más completa y pertinente: Telefónica, Sindicatos y Asociaciones de prejubilados. Utilicé, además, datos, tablas y gráficos, relacionados con la situación que estaba estudiando, publicados por organismos oficiales como el Ministerio de Trabajo y Asuntos Sociales, el Instituto Nacional de Estadística, el Centro de Estudios Sociales, Eurostat, etc. Otra fuente que consideré imprescindible fue la revisión de algunas investigaciones que tenían algún punto en común, Borderías (1993) y Del Bono (2002) por desarrollarse en el ámbito de Telefónica.

El trabajo de campo se llevó a cabo en Madrid. Si bien es cierto que Telefónica es una empresa que desarrolla su actividad en toda España y tiene empleados en todas las provincias, es esta ciudad la que cuenta con mayor número de ellos y, por tanto, la más afectada por los ajustes de plantilla. Revisando datos del ERE 2003-2007, se constata lo siguiente: en el Proyecto 2003, de las 5.384 solicitudes de baja, 1.428 correspondieron a Madrid, en el Proyecto 2004, de las 2.360 bajas, 623 fueron solicitadas en esta ciudad y en el Proyecto 2005, de las 712 solicitudes de baja presentadas hasta el 30 de marzo, 173 se cursaron en Madrid.

\section{Análisis y discusión de los resultados}

\section{Antecedentes}

Telefónica comenzó a desplegar su plan de reducción de plantilla a partir de 1993. Hasta ese momento el número de empleados de la empresa había ido en aumento, había pasado de 63.611, en 1987, a 74.340, en diciembre de 1993. En el Convenio Colectivo 1993-1995, firmado en marzo de 1994, se otorgó la posibilidad de jubilación anticipada voluntaria a partir de los 60 años, con abono por parte de la empresa del Convenio Especial con la Seguridad Social y con incentivos en función de la edad del trabajador que decidiese acogerse (a menor edad, mayor incentivo). Con el Plan Estratégico 1995-1999 se puso en marcha un programa de prejubilaciones para empleados de 58 y 59 años, manteniéndose el de jubilaciones anticipadas desde los 60 años. Además se planteó, por primera vez, la propuesta de bajas incentivadas para menores de 58 años. En el Convenio Colectivo de 1996 se ofrecieron jubilaciones anticipadas para los empleados desde los 57 años y bajas incentivadas para los menores de esta edad. Telefónica concluyó el ejercicio de 1996 con una plantilla de 67.217 empleados. El Convenio Colectivo 1997-1998 ofrece prejubilaciones desde los 55 años. Con el Plan de Adecuación de Plantillas 1998-2000, Telefónica pretendía quedarse con 20.000 empleados menos. Se mantuvieron las prejubilaciones desde los 55 años y las jubilaciones anticipadas desde los 60. Se estableció, además, un nuevo programa de excedencia de 1 a 5 años con posibilidad de incorporarse a los programas de pre-prejubilación, desde 50 a 54 años. Con el 
Convenio Colectivo 1999-2000 se firmó un ERE, el primero de Telefónica, que supuso la salida de 10.869 trabajadores. En el Convenio Colectivo 2001-2002 no hubo una nueva propuesta de ajuste, se siguió ejecutando la anterior. El día 23 de julio de 2003, Telefónica de España firmó con los representantes de los trabajadores el Convenio Colectivo 2003-2005, que incluye el ERE 44/03, segundo de su historia. La resolución del Expediente por el Ministerio de Trabajo y Asuntos Sociales se produjo con fecha 27 de julio de 2003 y autorizó a Telefónica de España S.A.U. a la extinción de los contratos de trabajo de 15.000 trabajadores de su plantilla, en el periodo 2003-2007.

\section{Cultura Telefónica}

Ser empleado de Telefónica genera (o al menos generaba) una identidad colectiva. Es la percepción que transmiten con claridad las personas que han trabajado en esta empresa, sobre todo si lo han hecho durante más de treinta años. Se tenía el sentimiento de que se vivía algo en común, de que se tenían intereses similares, expectativas parecidas, de que se había recorrido un largo camino común, el trabajador se sentía parte de una comunidad laboral. "Las culturas del trabajo son realidades claras, dinámicas que se construyen, cristalizan y modifican a través de un proceso histórico/temporal. El estudio diacrónico de los comportamientos sociales, actitudes y valores de un colectivo nos mostrará las diferentes fases o adecuaciones por las que su cultura del trabajo ha transitado" (Palenzuela, 1995:14). Estas palabras nos ayudan a comprender ese sentimiento de identidad colectiva que vivían los empleados de Telefónica, nos llevan a reflexionar sobre la 'cultura telefónica'. En más de ochenta años de existencia de Telefónica sus trabajadores habían ido interiorizando y expresando determinados comportamientos sociales, actitudes y valores. Comenzar a trabajar en Telefónica era entrar a formar parte de un colectivo que manifestaba una determinada identidad, la 'familia telefónica'. A la vez que se desarrollaba una actividad laboral el trabajador iba interiorizando los elementos que conformaban esa 'cultura telefónica'. Es un claro ejemplo de 'cultura del trabajo', de 'cultura obrera', es el conjunto de percepciones, sentimientos, actitudes, valores, representaciones, comportamientos, creencias, que las personas que trabajan o han trabajado en Telefónica han vivido, interiorizado, sentido, defendido, etc.

\section{Un trabajo para toda la vida}

Hace treinta, cuarenta años, cuando mis informantes firmaron un contrato de trabajo con Telefónica, daban por seguro que terminarían en la misma empresa, era el inicio de su trayectoria laboral. Telefónica era una empresa prestigiosa en la que se podía promocionar, incluso sin necesidad de tener mucha formación. La gran variedad de actividades desarrolladas, el gran número de empleados con que contaba y el hecho de que estuviera presente en toda la geografía española, facilitaba la promoción interna de los trabajadores. Los beneficios sociales eran muchos y el salario alto, si se comparaba con empresas del entorno, esa era la idea y así lo fueron constatando los empleados. Era, en definitiva, tener 'un trabajo para toda la vida', de ahí saldrían sólo cuando se jubilaran. 
La aceleración del cambio tecnológico, registrada a partir de la década de los 70, afectó especialmente al sector de las telecomunicaciones. En los años 90 Telefónica opta por la externalización y tercerización de servicios, la solución es el ajuste de plantilla y lo hace deshaciéndose del personal de más edad y muchos derechos adquiridos y contratando menor número de trabajadores, jóvenes, en ocasiones más formados y con salarios mucho más bajos. Nos encontramos ante la desvalorización de la antigüedad, lo que antes era un beneficio se convierte en un perjuicio. El ambiente laboral se deteriora. Este informante había salido de la empresa hacía cinco años, con 56 cumplidos y con treinta de servicio.

\footnotetext{
La decisión fue un poco obra de la empresa, porque ya la empresa nos... ${ }^{10}$ nos iba machacando poco a poco. (...) El ambiente en el trabajo no era nada bueno, porque estaban un grupo, que éramos veintidós empleados de... de Telefónica y en poco tiempo nos quedamos, de los veintidós nos quedamos dos nada más, y el resto se fue supliendo con... con gente de fuera, o sea con gente contratada ${ }^{11}$, entonces pues claro, pues no... no era el mismo ambiente, no... no estaba a gusto. $(\mathrm{E}-11)^{12}$
}

Una de las tácticas de presión utilizadas era dejar al trabajador sin actividad, sin trabajo real que hacer. Es un ejemplo más de deterioro del ambiente laboral que afecta a los más mayores. Con 51 años, y después de 33 desempeñando una función que le satisfacía: "Estaba harto de trabajar..., trabajar... Yo antes, cuando trabajaba, estaba de conductor y me quitaron. Y llevaba dos años que no tenía destino, cosa que yo no sabía qué hacer, que yo no..., yo no lo resistía, claro. En el momento que vi una oportunidad me fui, no me lo pensé dos veces y me fui." (E-7)

Cuando, en los años sesenta y setenta, comenzó su trayectoria laboral, mis informantes contaban con que tenían un trabajo para toda la vida y hasta que se jubilaran. Todos ellos, a la edad de entre 47 y 58 años, han cotizado entre 30 y 38. La primera parte de su sueño se ha cumplido, ese trabajo llenó toda su vida laboral ya que no desarrollarán, al menos en la mayoría de los casos, otro trabajo remunerado. Sin embargo, con lo que no contaban era con una interrupción tan temprana, entre 7 y 18 años antes de la edad legal de jubilación.

\section{La decisión}

El trabajo, el tiempo que se dedica al trabajo remunerado, ocupa una parte importante de la existencia social del individuo y de los grupos, lo hemos constatado en las respuestas dadas por los informantes. El conjunto de valores, representaciones y percepciones que los individuos interiorizan en su actividad laboral ayudan a mantener y reforzar la red social de la que forman parte. La centralidad del trabajo en la vida social, según P. Palenzuela (1995), se sustenta en el doble plano de su funcionalidad material e ideática. Todos mis informantes manifestaron que fue difícil tomar la decisión de interrumpir su vida laboral. El empleo, el trabajo remunerado, era una parte importante de su vida y lo había sido durante más de treinta años para todos ellos. El abandono de dicha actividad,

\footnotetext{
${ }^{10}$ Los puntos suspensivos, en las transcripciones de las entrevistas a los informantes, indican silencios, pausas prolongadas.

${ }^{11}$ Subcontrata de servicios a otras empresas.

${ }^{12}$ Utilizaré esta denominación, desde E-1 hasta E-23, para referirme a los informantes. Todas las entrevistas fueron llevadas a cabo entre los meses de enero y abril de 2005 , grabadas y posteriormente transcritas.
} 
la interrupción de la trayectoria laboral de forma temprana, no es una situación elegida, viene marcada por diversos factores, dependiendo de las características individuales de los afectados, tanto personales como relacionadas con el desempeño.

En el Plan Social 2003-2007, se dice expresamente “... procesos basados en los principios de voluntariedad, universalidad y no discriminación..." (Plan Social, 2003:2) Sin embargo es manifiesto que hay presiones, algunas personales, directas, sobre todo en el caso de los mandos y más si están 'fuera de convenio', lo hemos constatado con las declaraciones de los informantes. Estas presiones son las estrategias que utiliza la empresa, ya sean explícitas o implícitas, para conseguir el mayor número posible de renuncias al puesto de trabajo. La mayoría de ellas son implícitas y sirven para todos los trabajadores: rumores, pérdida de actividad, vacío de contenido del puesto de trabajo, deterioro del ambiente laboral, cambio de departamento, negativa a un traslado, etc. Esta informante lo expresa con claridad "No me he sentido presionada por la empresa, aunque bueno, es una voluntariedad un poco establecida y pactada." (E-13). Las que inciden exclusivamente en los mandos están relacionadas con la reducción o eliminación de sus competencias o del personal a su cargo: se les niegan traslados, se les amenaza con la pérdida del cargo y, por supuesto, de la gratificación que éste conlleva. Si son mandos fuera de convenio la presión es aún mayor, nombran en su lugar a personas más jóvenes, desaparecen sus competencias, se les relega del mando, etc. Se constata que, en todos los casos, estas presiones van dirigidas, sobre todo, a las personas de más edad, oficialmente desde los 52 años, aunque en algunos casos a los 49 o antes. La antigüedad dejó de ser un privilegio hace ya un tiempo. Otras presiones son objetivas, son generales y establecidas, son las estrategias explícitas que utiliza la empresa. Consisten en penalizaciones en la indemnización, un 5 $\%$ del salario regulador, por cada período de entre un día y seis meses de retraso en la decisión de acogerse al ERE, según la fecha que por edad corresponda a cada trabajador.

\section{Primeros momentos}

Me pareció importante ver cómo expresaban los informantes la sensación que vivieron los primeros días de su nueva situación. Conseguí un abanico muy amplio de términos. Se sentían 'como de vacaciones permanentes', 'tener días libres', 'no tener que madrugar', 'despistado', 'vértigo', 'raro', 'en las nubes', 'desconcertado', 'muy mal', 'cometiendo un pecado', 'con una sensación horrorosa de que perdía el tiempo', 'como cuando saltas de un trampolín', ... Después de una trayectoria laboral de entre 30 y 38 años, es lógico que el cese de la actividad remunerada genere sentimientos y vivencias importantes y a veces contradictorios. En unos casos eran positivos, relacionados con el descanso, el relax:

Al principio y, yo creo que todavía, tengo la sensación, la he tenido durante mucho tiempo, ahora ya se me va olvidando, pero, como que estaba de 'vacaciones permanentes', o sea, como que estoy de vacaciones. Y ¿cuándo vuelves a trabajar?, pues no sé, yo estoy de vacaciones, yo estoy de vacaciones, pues que sigo de vacaciones (risas), sigo de vacaciones. Entonces, cuando te vas de vacaciones sabes que tienes que volver y aquí pues, de momento, sigo sin volver (risas). O sea que es esa, es esa sensación, todavía un poco, que ya se me va olvidando un poco, ¿no? Porque ya vas llenando tus días con una actividad, más o menos, o sea vas cogiendo una rutina de 
hacer cosas y demás y entonces pues ya te das cuenta de que claro ya no estás de vacaciones, estás haciendo lo que te apetece, digamos. (E-2)

En otros, la vivencia era de desconcierto: "Había días que te sentías un tanto desconcertado. Incluso recuerdo... recuerdo alguna noche, a pesar de tenerlo totalmente claro, recuerdo alguna noche de... de soñar y levantarte un tanto... un tanto desconcertado, en el sentido de que no sabías si..., si..., si..., si te quedabas sin trabajo o qué podía pasar y tal..." (E-21). O de 'despiste': "Al principio un poco despistada, porque no sabía si estaba de vacaciones o ya no tenía que volver a trabajar." (E-9). "Sales de allí y estás unos días un poco que no sabes, un poco 'en las nubes'." (E-4).

Otros se sintieron mal: "Pues al principio me sentí muy mal." (E-15), tuvieron sensación de 'vértigo': "Sí, en el momento de irte un poco de vértigo, porque debe ser lo mismo a cuando saltas desde un trampolín, ¿no? Pues me preguntaba, cómo me voy a encontrar, qué voy a hacer en casa, cómo me voy a sentir, voy a echar de menos a estos impresentables de mis amigos y compañeros, y tal..." (E22). O lo vivieron incluso de forma traumática: "Al principio como que estaba cometiendo un pecado. Porque, no... (risas). ¡Ay Dios mío!, ¡tanto tiempo libre! Y yo qué hago. Era una sensación horrorosa de que perdía todo mi tiempo." (E-3).

La interrupción de una trayectoria laboral de más de treinta años, treinta y ocho en algunos casos, fue impactante para los entrevistados. Supuso la necesidad de adaptación a una nueva realidad. Se puede decir que consideraban el trabajo como una necesidad para su integración social. En algunos casos se constata la siguiente afirmación "El trabajo es más que trabajo: cuando desaparece, corren el riesgo de fracasar los modos de socialización vinculados a él y las formas de integración que él nutre". (Castel, 1995: 214). En otros casos, no muchos, aparecen otros modos de socialización ajenos al entorno laboral, o se desarrollan más algunos que ya existían. ¿Cuáles son?, lo vemos en los siguientes apartados.

\section{Ocupación del 'nuevo tiempo libre'}

En la mayoría de los casos, los informantes, desde que dejaron su trabajo remunerado, desde que su trayectoria laboral se vio interrumpida, no se dedican a actividades nuevas, intensifican, eso sí, sus relaciones familiares y de amistad y sus actividades de ocio: cine, lectura, paseos, museos, conferencias, senderismo, viajes, música, fotografía, deportes, gimnasia, baile, etc. "Hago lo que hacía antes, le dedico más tiempo. Pinto, coso, porque me encanta, me encanta. Pero también aprovecho para ir más al cine, salir con mis amigas, todos los días camino más de una hora..." (E-14). Tampoco tenían, en general, un plan de actividades previo a la salida de la empresa; en la mayoría de los casos en los que sí lo tenían, lo han desechado porque no llegaban a cumplirlo: "No me he organizado todavía ${ }^{13}$. He estado de..., de viajes y lo único que he hecho ha sido leer, pasear, hablar, estar con mi familia. (...) Sí, había hecho un plan, hacer alguna actividad en la Cruz Roja y, por supuesto, hacer ejercicio, ir a... a algún..., a algún gimnasio." (E-6). La sensación de falta de tiempo

\footnotetext{
${ }^{13}$ Hacía dos años que había salido de la empresa.
}

(c) Ma Victoria Inmaculada Velasco Juez. Publicado en AIBR. Revista de Antropología Iberoamericana, Ed. Electrónica Vol 1. Num. 3. Agosto-Diciembre 2006. Pp. 465-486 Madrid: Antropólogos Iberoamericanos en Red. ISSN: 1578-9705 
es también generalizada, no llegan a 'ver' las horas que antes dedicaban a Telefónica. "A nada, a nada, te he dicho que, en ese sentido, no, no me dedico a nada. (...) Un año sin trabajar y yo qué he hecho, no hice nada. Sí, bueno, termina el primer año y llega el segundo. Y el segundo pues vuelvo otra vez a la misma rutina, mi casa, ir a echar una mano a mi madre..." (E-5).

Excepto los mandos fuera de convenio, esto es lo que opina uno de ellos "Parece absurdo, cuando estabas trabajando, que pudieses echar de menos trabajar. No es que eche de menos trabajar, sino de hacer una actividad que... que a uno le guste." (E-17); todos los informantes reconocen haber encontrado actividades en las que canalizar el tiempo que antes dedicaban a su trabajo en Telefónica. En algunos casos, los menos, siempre mujeres, dicen que no hacen nada, cuando en realidad lo que hacen es intensificar el tiempo que dedican a las tareas del hogar. En general lo dedican a actividades de ocio y tiempo libre, a deportes, a reforzar las relaciones familiares, de amistad y de vecindad. Este es un ejemplo que nos permite, cuanto menos, dudar de la idea de Beck (1999: 69), de que la identidad y el estatus sociales sólo se consiguen a través del trabajo convencional y una buena profesión.

\section{Viejas relaciones, nuevos lazos}

Una de mis inquietudes a la hora de plantear la investigación tenía que ver con el ámbito relacional. P. Bouffartigue (1996: 97) en su reflexión sobre la pérdida de referencias sociales en relación con el trabajo, defiende que la descomposición del vínculo social es especialmente visible en los 'excluídos', en las personas sin empleo. También R. Castel (1995: 416) defiende la importancia del trabajo asalariado como fuente de identidad personal e integración social: con la precarización del empleo que conlleva el paradigma de la flexibilidad se corre el riesgo de perder el capital relacional. Sin embargo, mis informantes manifiestan, en general, que desde que interrumpieron su actividad laboral remunerada han intensificado su red de relaciones. En algunos casos se han visto obligados a reorientar su relación de pareja:

\footnotetext{
En alguna manera sí que han cambiado los encuentros con la pareja. Ves más a tu pareja y ha habido que matizarlos. Las relaciones eran, sobre todo, en vacaciones. Llega un momento en que te encuentras que tienes que convivir con una persona, que casi desconocías y no es exactamente lo que creías. Hay muchos matices que hay que conocerlos y hay que conseguir solucionar un poquito la cosa y convivir. Pero en estos 16 meses yo creo que hemos solucionado alguna cosa que ha podido haber y se ha centrado la relación. (E-12)
}

Han revisado la relación con sus hijos: "Y con mi hijo, pues..., pues que estoy más tiempo con él y bueno pues, a veces, sale un poco harto, lógico, el ya... el ya necesita su espacio." (E-16). "Que soy una pesada, que tengo mucho tiempo. Claro que han estado acostumbrados toda la vida a vernos poco y ahora pues tengo mucho tiempo para ellos y ellos dicen que no, que nos vayamos y les dejemos en paz." (E-9). Y con sus progenitores se hace más necesaria, debido a la edad: "Hay que dedicar una parte importante del tiempo a la familia, la familia porque tenemos progenitores muy longevos (...) y hay que dedicarles bastante tiempo." (E-12). 
En las relaciones sociales sobre todo se habla de amistad, del mantenimiento y afianzamiento de los lazos existentes, del inicio de algunos nuevos y del cese, en la mayoría de los casos, de la relación con colegas de trabajo, de aquellas que no se habían transformado en relación de amistad.

\section{La salud}

La mayoría de los informantes consideran que la nueva situación influyó positivamente en su salud. Reconocen una mejoría en su salud física y emocional por el hecho de haber dejado su trabajo en Telefónica. Se sienten más relajados, más tranquilos. "Ahora me dedico más a cuidarme, tanto en el aspecto físico, como en el aspecto psicológico, porque lógicamente, el hacer ejercicio te hace estar más relajada y te hace estar de otra manera, mejor." (E-2). Su tiempo es para ellos y el hecho de no tener horario es considerado positivo, "No he tenido ninguna época que me haya deprimido, ni que me haya afectado el haber dejado el trabajo. No, al contrario, pues he estado más relajada, más tranquila y eso también se nota, el no tener los horarios y las horas de trabajo. No hay problema de los turnos." (E-4)

Sin embargo, hubo algunos casos de deterioro manifiesto en la salud, todos ellos relacionados con las variables género y cargo ocupado en la empresa. Tres mujeres necesitaron tratamiento psiquiátrico durante unos meses. Consideran que el desencadenante fue la falta de cumplimiento de sus expectativas. Superaron el problema y en la actualidad se sienten bien. Pero el efecto más negativo en este ámbito lo viven los mandos fuera de convenio. Los tres entrevistados que pertenecen a esta categoría reconocen que el abandono de su actividad laboral ha influido mucho en su salud emocional:

\footnotetext{
A nivel emocional yo creo que sí, mucho, yo creo que sí influye, porque son muchas horas las que no haces nada. Y acostumbrada a un ritmo bestial, que traía yo, pues sí, yo creo que emocionalmente influye. Y a veces te das cuenta, que dices, vaya vida que llevo, ¿no? (...) Hay veces que..., que no..., que te encuentras que... que no has hecho nada, que has visto pasar el tiempo. (E-18)
}

Quizá la explicación esté en la pérdida de prestigio y reconocimiento social que conlleva el cese en el desempeño de un trabajo remunerado como el que ellos desarrollaban. Los tres eran mandos, jefes, tenían personal a su cargo, dirigían un departamento o servicio, tenían un elevado grado de responsabilidad y su dedicación era plena, es decir, al estar fuera de convenio su disponibilidad era total. Será difícil que puedan encontrar un sustitutivo, con actividades sociales o de ocio, al prestigio y estatus que les proporcionaba el puesto de trabajo que desempeñaban.

\section{Conclusiones y reflexiones}

Después de un exhaustivo análisis de la información obtenida de los actores objeto de esta investigación y partiendo de la idea de que la actividad laboral remunerada es un factor de identidad personal y social, un factor de integración y estructuración del ser humano, se puede concluir que, desde una perspectiva antropológico-social, la desvinculación temprana del trabajo remunerado 
conlleva la necesidad de ajuste en todas las actividades del ser humano, tanto en sus relaciones familiares (pareja, hijos, progenitores) y sociales, como en la utilización de su tiempo e incluso en su salud. El alcance, la orientación y las consecuencias de estos efectos es lo que hemos visto con esta investigación. He tratado de identificar y describir cómo viven, cómo sienten, cómo valoran y cómo canalizan estos actores, el colectivo de trabajadores de Telefónica que he estudiado, los efectos de su desvinculación de la actividad laboral.

La interrupción anticipada de una trayectoria laboral de más de treinta años es un hecho, aunque a veces esperado, en muy pocas ocasiones deseado. Puede ser esperado por la situación del entorno, tanto social como de la propia empresa. Hemos visto cuáles han sido los cambios que se han ido produciendo en los últimos quince años en el mundo del trabajo y las consecuencias que éstos han ocasionado en los recursos humanos de las empresas.

Cuando, en 1993, Telefónica llevó a cabo su primer plan de reestructuración de plantilla, todo empezó a desmoronarse. A partir de ahí no ha habido tregua para los trabajadores. En ese año, como hemos visto anteriormente y repito por la importancia que tiene, Telefónica de España contaba con 74.340 empleados y pretende cerrar el ejercicio de 2007 con 25.650. En 14 años tendrá lugar una reducción de plantilla de más del $66 \%$, casi 50.000 puestos de trabajo destruidos. Desde entonces este grupo de actores vivió sus últimos años de asalariados, con una relativa incertidumbre ante el futuro, con la idea de que su trayectoria laboral terminaría antes de cumplir la edad legal de jubilación. Era un hecho asumido. Ninguno de ellos se planteaba siquiera tener que trabajar hasta los 65 años, quizá por el hecho de que en Telefónica, y gracias a la Institución Telefónica de Previsión (ITP) ${ }^{14}$, todos los trabajadores podían jubilarse a los 60 años, sin ningún tipo de penalización. Lo que no tenían claro, sin embargo, era la edad a la que ese hecho se produciría. En general tenían la esperanza de que ocurriera a una edad más avanzada, más cercana a los sesenta años. La realidad era otra, a medida que se firmaban los sucesivos Convenios Colectivos la edad se adelantaba y las condiciones empeoraban. Este hecho generó cierta 'presión' en los afectados, por un lado no querían perder la oportunidad de 'librarse' de un cierto número de años de actividad laboral, además la compensación económica cada vez era más reducida y, por otro lado, sentían que eran muchos los años en los que acortarían su trayectoria laboral, mucho el tiempo en el que tendrían que buscarse otra ocupación, mayor la necesidad de ajuste en su vida.

Los trabajadores justifican su decisión de acogerse a la desvinculación anticipada de muchas maneras, argumentos no les faltan. Pueden y deben ser capaces de transformar una situación negativa en algo positivo. De hecho, en muchos casos, lo hacen. La decisión, aunque documentalmente es voluntaria ${ }^{15}$, incluso aunque la mayoría de los afectados y afectadas dicen que

\footnotetext{
${ }^{14}$ La ITP fue creada en 1944 en sustitución de la Institución Benéfica de Previsión. En su sostenimiento participaban trabajadores y empresa. Cubría la jubilación anticipada hasta los 65 años, sin ningún tipo de penalización, de los trabajadores que quisieran jubilarse desde los 60. Fue disuelta en 1988 con el consiguiente perjuicio para los trabajadores que habían cotizado durante toda su vida laboral en Telefónica.

15 "... existiendo la necesidad de conseguir la adecuación de la plantilla" (...) "se articulan varios procesos basados en los principios de voluntariedad, universalidad y no discriminación..." (Plan Social, 2003: 2).
} 
no se han sentido presionados, no es libre. Son muchos los motivos que empujan a los trabajadores a tomar la decisión de dejar la empresa, de dar por finalizada su trayectoria laboral, ha sido ampliamente expresado por los informantes.

Un primer grupo de estos motivos están relacionados con la propia empresa, con las estrategias de presión que utiliza, unas implícitas y otras explícitas. Entre ellas se puede considerar la transformación que ha sufrido Telefónica en los últimos quince años, con los sucesivos y reiterados anuncios, hechos realidad, de la necesidad de reducir la plantilla; la externalización de servicios, con la consiguiente creación de empresas del grupo, de características, en cuanto a derechos de los empleados, totalmente alejadas de lo que los 'telefónicos' vivían como 'la cultura telefónica'; el deterioro que se vivía en el ambiente laboral, no se sabía hacia dónde iba la empresa, los colegas eran cada vez menos 'telefónicos' y más trabajadores de las empresas del grupo; la sensación de alejamiento que vivían los empleados, en relación con los objetivos de la empresa; el cambio de visión en cuanto a la consideración del cliente, la consigna hace unos años era 'el cliente siempre tiene razón', en la actualidad, la opinión de los empleados era que la empresa se mantenía no porque su gestión fuera excelente sino porque 'las demás lo hacían peor'; los rumores de empeoramiento de la situación de la empresa, de que sería el último ERE, de que si no se acogían a él el número de trabajadores previsto los despidos serán forzosos; etc.

Telefónica, en el Plan Social 2003-2007, justifica la puesta en marcha del Expediente de Regulación de Empleo por las causas motivadoras que "se enmarcan en un contexto general del entorno macroeconómico, que afecta de manera importante al sector en el que actúa Telefónica de España S.A.U." (Plan Social, 2003: 2) Entre ellas señala una importante desaceleración económica en los países de la Unión Europea, que está afectando al sector básico de la economía española, principalmente al sector de las Telecomunicaciones. En el citado Plan Social constan las estrategias o presiones explícitas, las penalizaciones que se aplicarán al trabajador que no se acoja a la situación propuesta en la fecha que le corresponda por edad.

Otro argumento expresado por los informantes, que influyó a la hora de tomar la decisión, fue el panorama laboral de nuestro país en los últimos años. Se piensa en la elevada tasa de desempleo y la pregunta de los afectados es ¿pasaremos a engrosar ese número? En cuanto a la contratación precaria no se puede dejar de plantear la posibilidad de que la empresa decida que algunos trabajadores pasen a las filiales, a las empresas del grupo, con la consiguiente pérdida de derechos adquiridos y antigüedad. También se tiene en cuenta que cada vez hay más pensionistas en nuestro país y que, desde hace años, se comenta que hay un gran déficit en las arcas del Estado en relación con la partida presupuestaria dedicada al pago de las pensiones. ¿Quedará algo para cuando nos jubilemos?, ¿podremos cobrar una pensión digna? Fueron comentarios y preguntas planteados, aunque no en las entrevistas individuales, sí en los grupos de discusión, que se prestaron más a consideraciones genéricas. Estas situaciones eran conocidas y comentadas por los trabajadores. En otra época, unos años antes, se veían lejanas, afectaban a 'otros' trabajadores, podían afectar a los 
hijos de los empleados pero no a los propios empleados de Telefónica, quienes tenían un puesto de trabajo fijo y seguro, en una empresa que aumentaba más y más su plantilla.

Había un tercer grupo de motivos que son más personales, más subjetivos, están relacionados con las características de cada afectado o afectada, ya sean personales, familiares o profesionales. Como he comentado, la mayoría de los informantes no reconocen, abiertamente, presiones. En algunos casos llevan diez años haciéndose a la idea y asumiendo que dejarían de trabajar a una edad temprana. Sin embargo, en ocasiones, la propuesta de la empresa les 'ha pillado desprevenidos'..., pensaban que podrían quedarse un poco más de tiempo, pero la decisión ha de ser tomada de forma rápida. No hay mucho tiempo para pensar. Si no aceptan cuando se les propone, quizá nunca más se les presente la oportunidad y, si se presenta, será en peores condiciones.

Las hipótesis planteadas en la investigación relacionaban las categorías: género, mando (dentro o fuera convenio)/no mando, si la persona tiene o no cargas familiares, la organización de su tiempo, el ámbito relacional, la salud, etc. En mayor o menor medida todas ellas se cumplen. Sin embargo, la que queda demostrada con más fuerza es la relativa a la categoría 'mando/no mando', sobre todo a los mandos fuera de convenio, a los trabajadores que ocupaban en la empresa un cargo no regulado por un Convenio Colectivo. En estos casos, la actividad que desarrollaban conllevaba la dedicación plena de su tiempo y les confería un estatus, prestigio y reconocimiento social importantes, tanto interno como externo. Entre familiares, amigos y vecinos dejar de desempeñar un cargo en Telefónica supone una pérdida de estatus, sin embargo la mayor compensación económica ayuda a mantenerlo. No ocurre lo mismo con el reconocimiento social interno, cuya pérdida es total, dejan de tener subordinados, trabajadores a quienes mandar y ese vacío es muy importante. Los actores, del grupo de informantes, que cumplen esta variable, tanto varones como mujeres, a pesar de que hace entre dos y siete años que se habían desvinculado, siguen pensando en la necesidad de llenar su tiempo con otra actividad laboral remunerada. No se acostumbran a la nueva situación social, es difícil que encuentren otras actividades que llenen ese vacío relacional de estatus y prestigio. Todos ellos manifiestan que su salud emocional se ha visto afectada.

Otra de las categorías en las que he encontrado algún efecto significativo es el género cuando se cruza con la salud. Hay un grupo de actores, mujeres en este caso, una independiente que vive con una hermana; las otras tres con hijos mayores, aunque todavía formando parte del núcleo familiar, dos de ellas con pareja (uno en activo y el otro también desvinculado) y la otra separada, que también manifiestan haber pasado por una situación negativa en cuanto a su salud emocional. El origen del problema estuvo en que cuando dejaron la empresa no consiguieron ocupar su tiempo de forma satisfactoria, sus expectativas no se vieron cubiertas. Tres de ellas siguieron un tratamiento psiquiátrico durante unos meses que les ayudó a superar la situación y a encauzar su vida, a ocupar su tiempo de forma más grata. En la actualidad se encuentran bien y expresan su satisfacción por el giro, en positivo, que ha dado su vida. El resto de los informantes consideran que la nueva situación de desvinculados de la actividad laboral remunerada ha influido en una mejora de su salud emocional 
y física. Ninguno de los actores considera que su salud física se haya visto afectada de forma negativa. Si han vivido algún cambio en este sentido, piensan que es debido a la edad o que es un hecho fortuito.

Los varones desvinculados, cuando su pareja sigue en activo, reconocen que asumen algunas tareas más en el hogar, pero no muchas, sobre todo porque la mujer sigue asumiendo el rol de esposa, madre y ama de casa, a la vez que el de asalariada. Los informantes, sobre todo mujeres con hijos, reconocen que dedican más tiempo a las relaciones familiares, tanto a los hijos como a los padres. También algunos varones dedican más tiempo a sus progenitores. ¿Qué hay de la consideración del desarrollo de las tareas del hogar como trabajo?, las informantes no lo viven como tal, siguen considerándolo como su actividad principal, como algo consustancial a su rol de mujer, esposa y madre. El desempeño de una actividad laboral remunerada ha sido como un paréntesis en su vida, cuando ese trabajo termina, siguen teniendo el suyo, el de toda la vida, el que les 'corresponde'.

Las parejas, cuyos dos miembros están desvinculados, en general, consideran que tienen que reaprender a convivir. El hecho de que cuenten con mucho más tiempo para compartir hace que tengan que replantearse la relación. Es un reencuentro con la persona con quien han compartido los últimos 25, 30 ó 35 años, a quien se puede llegar a ver como extraña. Se constata con casos de divorcios y separaciones conocidos por algunos de los informantes.

La mayoría de afectados no habían hecho un plan de actividades, no habían decidido en qué iban a emplear el tiempo que Telefónica les iba a dejar libre. Quienes sí lo habían hecho manifiestan que lo han desechado porque no lo cumplían. Las actividades que se llevan a cabo son las que van surgiendo día a día. En general se tiene la idea de que el tiempo no cunde, no se hacen tantas cosas como gustaría. Lo que sí se cumple, y es constatable, es que aquellas personas que, antes de abandonar la actividad laboral remunerada, llevaban a cabo determinadas actividades de ocio, ahora las realizan con más intensidad, les dedican más tiempo.

La reducción en la remuneración económica también es un hecho patente y tenido en cuenta por los actores, sobre todo a medida que pasan los años en la nueva situación de desvinculados. Sin embargo, la mayoría de los informantes reconocen que tienen suficiente para vivir y que el hecho de contar con más tiempo para ellos, para hacer lo que les gusta, les compensa suficientemente. Solamente en el caso de la informante que negoció un despido improcedente con la empresa la situación es vista como muy negativa, porque ha de seguir cotizando a la Seguridad Social, en este caso por su cuenta, si quiere tener derecho a una jubilación plena cuando cumpla la edad legal.

El sentimiento general de los actores, ante la nueva situación que viven, es de libertad, de bienestar, de poder dedicar el tiempo a sí mismos, a lo que les gusta, a actividades más gratificantes. No tienen que cumplir un horario, no tienen que madrugar, no tienen un jefe, tienen más tiempo libre y tienen un salario. R. Sennett (2000:124) defiende que las reducciones de plantilla, llevadas a cabo por las 
empresas, pueden generar un sentimiento de fracaso e influir en el carácter de los trabajadores. Después de analizar las manifestaciones de los actores, veo que esta tesis se constata en los mandos fuera de convenio, es claro el sentimiento de fracaso y ellos mismos reconocen influencia en su carácter. En el resto de informantes, no se demuestra el sentimiento de fracaso, aunque se puede decir que su carácter se ha visto influido de alguna forma.

Hasta aquí, y a través de las vivencias y experiencias narradas por los informantes, he presentado la situación de Telefónica S.A.U., en relación con el empleo, en la actualidad. Las cosas son así, el problema está ahí y parece que la única solución es la que se está llevando a cabo. No sabemos si la empresa se ha planteado otras alternativas. Sí que sabemos, sin embargo, que hay autores que han reflexionado acerca del futuro de nuestra sociedad actual. En su informe para la OCDE sobre el futuro del trabajo, la familia y la sociedad en la Era de la Información, Carnoy y Castells (1997:23) definen con gran precisión el futuro que se nos avecina: "Lo que emerge de nuestro análisis es la visión de una economía extraordinariamente dinámica, flexible y productiva, junto con una sociedad inestable y frágil, y una creciente inseguridad individual". El problema de los trabajadores mayores, de su difícil inserción social debido a una trayectoria laboral rota, no será en el futuro, lo es cada vez menos, un problema asociado a la edad, sino un problema que acabará afectando a la mayoría de la sociedad, independientemente de la edad.

Algunos autores han propuesto alternativas a esta situación, a estos 'despidos' masivos, al desempleo en general, a la flexibilidad precarizadora. Martin Carnoy y Manuel Castells (1997), proponen organizar redes que configuren itinerarios en torno a la educación, la formación profesional y la información. Con una visión diferente, O. Giarini y P. Liedtke, en su Informe al Club de Roma (1998), defienden un sistema de trabajo multiestratificado, de manera que se reconozcan tres estratos diferenciados de actividades productivas: el primero, un trabajo remunerado, equivalente a lo que puede ser el tiempo de trabajo básico, garantizado para todas las personas capaces, mediante la intervención pública; el segundo, el trabajo remunerado desarrollado en condiciones de mercado; y el tercero, las actividades de autoproducción, así como las voluntarias no remuneradas. Siguiendo esta línea, teniendo en cuenta otro tipo de actividades, además de las productivas, Paul Bouffartigue (1999) propuso la creación de un mecanismo de contratos de actividad, que relacione a cada persona con una red de empresarios, asociaciones y organismos de formación, de manera que cada individuo acumula unos derechos en la medida en que participa en las actividades de esa red, actividades que pueden ser laborales, sociales o formativas. A. Suso e I. Zubero (2002:40) defienden que "el desempleo de los mayores (y sin duda el del resto de grupos sociales) sólo encontrará solución utilizando un modo de pensar distinto del que es dominante en el sistema actual".

Es necesario, si queremos encontrar solución a la situación actual, replantear la relación que históricamente se ha mantenido con el mundo del trabajo. Nos enfrentamos a un problema que implica una ruptura en la evolución de nuestra sociedad. Una propuesta que me atrevo a plantear, y que probablemente sería compatible con algunas de las anteriores, es mayor flexibilidad en las 
excedencias, un mejor sistema de excedencias con garantía de respeto del puesto de trabajo. Imagino que sería posible también en otras empresas, pero en el caso de Telefónica, que es el que conozco, después de años de observación y participación en grupos informales de empleados, me permito decir que habría sido o podría ser una alternativa. No serviría ciertamente para evitar 15.000 despidos en cinco años y menos 50.000 en catorce años, pero sí para permitir a algunos trabajadores enriquecerse culturalmente con periodos de convivencia de una manera diferente, periodos en los que habrían podido mejorar su formación, su experiencia, sus habilidades. Si existiera la posibilidad de pedir una excedencia por motivos personales, sin tener que dar explicaciones, pienso que podría ser una forma de que algunos empleados se turnaran en un mismo puesto de trabajo. Me consta que entre los trabajadores hay muchas personas con inquietudes, tanto de aprendizaje como de aventura, de vivir nuevas experiencias, de compartir vivencias diferentes, con personas diferentes, en ámbitos diferentes, en culturas diferentes. Un año sabático, sin sueldo en este caso, con la única exigencia, que no es poca, del mantenimiento del puesto de trabajo en las mismas condiciones de actividad y lugar de residencia, sería una alternativa para un determinado número de trabajadores. Quizá no fuera una medida muy demandada, pero supondría una importante mejora social para las personas interesadas, cuya motivación aumentaría significativamente, y una mejora en el prestigio de la empresa. Esa sería mi propuesta a la Dirección de Recursos Humanos si fuera consultora de Telefónica.

En la actualidad existe la posibilidad, según recoge el Estatuto de los Trabajadores (Artículo 46. Epígrafe 3), de solicitar excedencia: "El trabajador con al menos una antigüedad en la empresa de un año tiene derecho a que se le reconozca la posibilidad de situarse en excedencia voluntaria por un plazo no menor a dos años y no mayor a cinco". Sólo si la excedencia es por maternidad, no hay un tiempo mínimo. Pienso que dos años es demasiado tiempo para poder vivir sin salario, desde el planteamiento que estoy haciendo. Por otra parte, tampoco está previsto que la empresa reserve el puesto de trabajo al empleado que sale en excedencia. Así queda expresado también en el Estatuto de los Trabajadores (Artículo 46. Epígrafe 5): "El trabajador excedente conserva sólo un derecho preferente al reingreso en las vacantes de igual o similar categoría a la suya que hubiera o se produjeran en la empresa". Hay empresas, entre ellas Telefónica, que van por delante del Estatuto de los Trabajadores, en algunos aspectos. ¿Por qué no en otros, como las excedencias?

\section{Referencias bibliográficas}

Bauman, Zygmunt (2003) [1998]. Trabajo, consumismo y nuevos pobres. Barcelona: Gedisa.

Beck, Ulrich (2000) [1999]. Un nuevo mundo feliz. La precariedad del trabajo en la era de la globalización. Barcelona: Paidós.

Borderías, Cristina (1993). Entre líneas. Trabajo e identidad femenina en la España Contemporánea La compañía Telefónica 1924-1980. Barcelona: Icaria.

(c) Ma Victoria Inmaculada Velasco Juez. Publicado en AIBR. Revista de Antropología Iberoamericana, Ed. Electrónica

Vol 1. Num. 3. Agosto-Diciembre 2006. Pp. 465-486 Madrid: Antropólogos Iberoamericanos en Red. ISSN: 1578-9705 
Bouffartigue, Paul (1996). ¿Fin del trabajo o crisis del trabajo asalariado? Sociología del Trabajo, (nueva época, 29, invierno 96/97):91-110.

Bouffartigue, Paul (1999). Francia: ¿la norma del empleo hecha trizas? En La crisis del empleo en Europa. Prieto, Carlos. 2 vols. Alzira: Germania.

Carnoy, Martin y Castells, Manuel (1997). Sustainable Flexibility. Paris: OCDE.

Castel, Robert (1997) [1995]. Las metamorfosis de la cuestión social. Una crónica del salariado. Barcelona: Paidós.

CES (2002). Informe sobre vida laboral y prejubilaciones. Madrid.

Cortes Generales (1978). Constitución Española de 27 de diciembre de 1978. B.O.E. 311 de 29/12/1978: 2931529424.

Cortes Generales del Estado (1997). Pactos de la Moncloa. B.O. de las Cortes, de 17 de noviembre de 1977. Número 32

Del Bono, Andrea (2002). Telefónica. Trabajo degradado en la era de la información. Madrid: Miño y Dávila.

Festinger, Leon (1975) [1957]. Teoría de la disonancia cognoscitiva. Madrid: Instituto de Estudios Políticos.

Giarini, Orio y Liedtke, Patrick M. (1998). El dilema del empleo. El futuro del trabajo. Informe al Club de Roma. Barcelona: Galaxia Gutenberg/Círculo de Lectores.

Jefatura del Estado (1980). Ley 8/1980, de 10 de marzo, del Estatuto de los Trabajadores. B.O.E., de 14/3/1980. Número 64: 5799-5815.

Jefatura del Estado (1998). Ley 47/1998, de 23 de diciembre, de Jubilación anticipada del sistema de la Seguridad Social. B.O.E., de 29/12/1998. Número 311: 43718-43719.

Ministerio de Trabajo y Asuntos Sociales (1994). Convenio Colectivo 1993-1995 de Telefónica de España, Sociedad Anónima. B.O.E., de 20/8/94. Número 199: 26734-26734.

Ministerio de Trabajo y Asuntos Sociales (1996). Convenio Colectivo 1996 de Telefónica de España, Sociedad Anónima. B.O.E., de 19/6/1996. Número 148: 20146-20161.

Ministerio de Trabajo y Asuntos Sociales (1997). Convenio Colectivo 1997-1998 de Telefónica de España, Sociedad Anónima. B.O.E., de 29/9/97. Número 233: 28415-28434.

Ministerio de Trabajo y Asuntos Sociales (1999). Convenio Colectivo 1999-2000 de Telefónica de España, Sociedad Anónima. B.O.E., de 10/12/1999. Número 295: 42713-42724.

(c) Ma Victoria Inmaculada Velasco Juez. Publicado en AIBR. Revista de Antropología Iberoamericana, Ed. Electrónica

Vol 1. Num. 3. Agosto-Diciembre 2006. Pp. 465-486 Madrid: Antropólogos Iberoamericanos en Red. ISSN: 1578-9705 
Ministerio de Trabajo y Asuntos Sociales (2001). Convenio Colectivo 2001-2002 de Telefónica de España, S.A.U. B.O.E., de 2/7/2001. Número 157: 13500-23521.

Ministerio de Trabajo y Asuntos Sociales (2003). Convenio Colectivo 2003-2005 de Telefónica de España, S.A.U. B.O.E., de 16/10/2003. Número 248: 37114-37132.

Palenzuela, Pablo (1995). Las culturas del trabajo: Una aproximación antropológica. Sociología del Trabajo, (nueva época, 24):3-28.

Prejubilados (2003). Expediente de Regulación de Empleo 2003-2007. Guía Sindical de consulta. UGT. En http://fetcm.ugt.org/comunicaciones/prejubilados/ere 2003/Guia\%20Sindical\%20ERE\%202003.pdf. Julio de 2003. Accedido el 3 de Abril de 2006.

Riera, José María (1999). Jubilarse a los 50. Madrid: Pirámide.

Rivas Rivas, Ana María (2005). Del trabajo como valor de inscripción social al trabajo como factor de desestructuración social. Cuadernos de Relaciones Laborales, 22(2):63-82.

Sennett, Richard (2000) [1998]. La corrosión del carácter. Las consecuencias personales del trabajo en el nuevo capitalismo. Barcelona: Anagrama.

Suso, Anabel y Zubero, Imanol (2002). Expulsados del trabajo... y más. Un estudio de la salida anticipada del mercado de trabajo de los trabajadores mayores. Sociología del Trabajo, 46 (nueva época, otoño 2002):19-44.

Telefónica de España, S. A. U. (1995). Plan Estratégico 1995-1999. Dirección General Adjunta de Recursos Humanos. 25 de enero de 1995.

Telefónica de España, S. A. U. (1998). Plan de Adecuación de Plantillas 1998-2000. Dirección General Adjunta de Recursos Humanos. Abril de 1998.

Telefónica de España, S. A. U. (2003). Plan Social 23/07/03, anexo al Convenio Colectivo 2003-2005. Dirección General Adjunta de Recursos Humanos. Julio de 2003.

Telefónica de España, S. A. U. (2005). Informe Anual 2004. Dirección General de Comunicación Corporativa. Mayo de 2005.

Zubero, Imanol (1996). Participación y democracia ante las nuevas tecnologías. Retos políticos de la sociedad de la información. Telos, 45:26-35. 